RAIRO Operations Research

RAIRO Oper. Res. 35 (2001) 301-313

\title{
EFFICIENT AND LOCAL EFFICIENT SOLUTIONS FOR ASSIGNMENT TYPE PROBLEMS*
}

\author{
JaCques A. Ferland ${ }^{1}$ And Pina Marziliano ${ }^{1}$
}

\begin{abstract}
In this paper, we analyse the multiobjective problem generated by applying a goal programming approach to deal with linear assignment type problem. We specify sufficient conditions for a solution to be efficient for this problem. The notion of efficiency with respect to a neighborhood is also introduced and characterized through sufficient conditions. Unfortunately, these conditions are not necessary in general.
\end{abstract}

Keywords: Efficiency, local efficiency, assignment,multiobjective.

\section{INTRODUCTION}

The structure of the Assignment Type Problem (ATP) provides a very interesting framework to model timetabling and scheduling problems (see [2]). In Ferland et al. [4], four different neighborhood search techniques are analyzed to deal with the single objective function optimization problem generated when a penalty approach is used to handle the additional constraints. In Ferland et al. [3], one of these techniques, the Tabu Search Method, is extended to deal with the multiobjective function optimization problem generated when a goal programming approach is used to deal with the additional constraints. The method generates an efficient solution of the multiobjective problem associated with a specific priority ranking of the objective functions. These techniques have been used in several applications as reviewed in Costa [1], Ferland [2], and Robert [12].

Received December, 1999. Accepted March, 2001

* This research was supported by NSERC grant OGP0008312, and FCAR grant ER-1654. The second author received a NSERC fellowship during her M.Sc. studies.

1 Département d'Informatique et de Recherche Opérationnelle, Université de Montréal, C.P. 6128, Succursale Centre-Ville, Montréal Qubec Canada H3C 3J7.

(c) EDP Sciences 2002 
The drawback of the preceding techniques is that they generate only one specific efficient solution. In Marziliano [9] and Marziliano and Ferland [10], a variant of the interactive method of Zionts and Wallenius [14] is introduced to generate sets of neighboring local efficient solutions to be appraised by the user. At each iteration, the feedback provided by the user is used to identify the set of local efficient solutions to be generated at the next iteration.

The purpose of this paper is to introduce formally the notion of local efficient solutions with respect to a neighborhood for linear ATP, and to provide sufficient conditions for efficiency and local efficiency. In Section 2, we give a mathematical formulation of ATP and we specify the goal programming approach to transform the problem into a multiobjective problem. Sufficient conditions for a solution to be efficient for this multiobjective problem associated with a linear ATP are introduced in Section 3. Then the notion of local efficiency with respect to a neighborhood for the same problem and sufficient conditions for it are given in Section 4. Unfortunately these conditions are not necessary in general. In Section 5, we establish the relations between the local efficiency conditions and the efficiency conditions. Finally, Section 6 includes concluding remarks extending these results to a generalized version of ATP.

\section{Problem Formulation AND BASiC DEFinitions}

Consider the Assignment Type Problem (ATP) that can be summarized as follows:

Given $n$ items and $m$ resources, the problem is to determine an assignment of each item to a resource in order to optimize an objective function and to satisfy $K$ additional constraints.

This problem can be formulated as

$$
\begin{array}{rc}
\min & F(x) \\
\text { Subject to } & G_{k}(x) \leq 0 \quad 1 \leq k \leq K \\
& x \in \mathcal{X}
\end{array}
$$

where

$$
\mathcal{X}=\left\{x: \sum_{j \in J_{i}} x_{i j}=1,1 \leq i \leq n ; x_{i j}=0 \text { or } 1,1 \leq i \leq n, j \in J_{i}\right\}
$$

and $J_{i} \subset\{1,2, \ldots, m\}$ is the set of admissible resources for item $i, 1 \leq i \leq n$. The decision variable $x_{i j}$ is such that

$$
x_{i j}= \begin{cases}1 & \text { if item } i \text { is assigned to resource } j \\ 0 & \text { otherwise. }\end{cases}
$$


For all $x \in \mathcal{X}$, denote by $V_{k}(x)$ the violation of constraint $G_{k}(x) \leq 0$; i.e.,

$$
V_{k}(x)=\operatorname{Max}\left\{0, G_{k}(x)\right\}, 1 \leq k \leq K .
$$

In most real world applications, the additional constraints $G_{k}(x) \leq 0,1 \leq k \leq K$, are regarded as goals to be achieved rather than constraints. Hence it makes sense to replace the original $(A T P)$ by a multiobjective programming problem

$$
(\mathrm{GATP}) \min _{x \in \mathcal{X}} V(x)=\left[V_{1}(x), V_{2}(x), \ldots, V_{K}(x), V_{K+1}(x)\right]^{T}
$$

where $V_{K+1}=F(x)$.

Recall that in multiobjective problems the notion of efficiency replaces the notion of optimality used in single objective problems:

$x \in \mathcal{X}$ is efficient for $(G A T P)$ if there is no $z \in \mathcal{X}$ such that

$$
\begin{aligned}
& V_{k}(z) \leq V_{k}(x) \quad 1 \leq k \leq K+1 \\
& V_{\bar{k}}(z)<V_{\bar{k}}(x) \quad \text { for some index } \bar{k}, 1 \leq \bar{k} \leq K+1 \text {. }
\end{aligned}
$$

Geoffrion [6] introduces a sufficient criterion for efficiency, allowing to identify efficient solutions by solving single objective function problems associated with $(G A T P)$ :

$\bar{x} \in \mathcal{X}$ is efficient for $(G A T P)$ if there exist positive weights

$$
\lambda_{k}>0,1 \leq k \leq K+1,
$$

such that $\bar{x}$ is an optimal solution of the associated weighted problem

$$
\left(\operatorname{GATP}_{\lambda}\right) \min _{x \in \mathcal{X}} \sum_{k=1}^{K+1} \lambda_{k} V_{k}(x) .
$$

It is worth noting that in several applications reported in Ferland [2], we use a penalty approach to deal with the underlying $(A T P)$. In this approach, the following problem $(P A T P)$ is solved instead of $(A T P)$ :

$$
\text { (PATP) } \min _{x \in \mathcal{X}} \sum_{k=1}^{K+1} \beta_{k} V_{k}(x) .
$$

Hence this corresponds to determine an efficient point of $(G A T P)$ associated with the weights $\beta_{k}>0,1 \leq k \leq K+1$.

In this paper, we consider the case where $F(x) \equiv 0$ and all functions $G_{k}(x)$ are linear. This is far from being an unrealistic case since in several applications the objective is to obtain a solution satisfying the additional constraints as much 
as possible without any objective function per se. For instance, in the school timetabling problem, the purpose is to generate a timetable with the least number of conflicts (where the same teacher or the same student must attempt more than one class at a given period), respecting teachers' availabilities, and where at each period, the number of required classrooms exceeds by as few as possible the number of classrooms available. Under these assumptions $(G A T P)$ reduces to

$(G A T P L)$

$$
\min _{x \in \mathcal{X}} V(x)=\left[V_{1}(x), V_{2}(x), \ldots, V_{K}(x)\right]^{T}
$$

where

$$
V_{k}(x)=\max \left\{0, \sum_{i=1}^{n} \sum_{j \in J_{i}} b_{i j}^{k} x_{i j}\right\}
$$

Furthermore, the associated weighted problem becomes

$$
\begin{aligned}
& \left(G A T P L_{\lambda}\right) \\
& \min \quad \sum_{k=1}^{K} \lambda_{k} s_{k} \\
& \text { subject to } \sum_{i=1}^{n} \sum_{j \in J_{i}} b_{i j}^{k} x_{i j}-s_{k}+t_{k}=0 \quad 1 \leq k \leq K \\
& \begin{array}{ll}
\sum_{j \in J_{i}} x_{i j}=1 & 1 \leq i \leq n \\
x_{i j}=0 \text { or } 1 & 1 \leq i \leq n, j \in J_{i} \\
s_{k} \geq 0, t_{k} \geq 0 & 1 \leq k \leq K .
\end{array}
\end{aligned}
$$

\section{EFFICIENCY CONDITIONS}

In this section, we introduce a sufficient condition for a solution $x \in \mathcal{X}$ to be an efficient point of $(G A T P L)$ associated with a weight vector $\lambda>0$. First, we introduce sufficient conditions for $x$ to be an optimal solution of $\left(G A T P L_{\lambda}\right)$ by referring to the optimality condition of the relaxed problem $\left(\overline{G A T P L}_{\lambda}\right)$ obtained by relaxing the integrality conditions on the variables $x_{i j}$; i.e. the constraints $x_{i j}=0$ or 1 are replaced by $x_{i j} \geq 0,1 \leq i \leq n, j \in J_{i}$. Then, relying on Geoffrion [6] result, it follows that $x$ is an efficient point of (GATPL).

Property 3.1. Consider problem $(G A T P L)$. Let $\lambda>0$ be a weight vector, and $\bar{x} \in \mathcal{X}$ be such that for all $i, 1 \leq i \leq n$

$$
\text { and } \begin{array}{cc}
\bar{x}_{i j(i)}=1 & \text { for some index } j(i) \in J_{i} \\
& \bar{x}_{i j}=0 \quad \text { for all } j \in J_{i}, j \neq j(i) .
\end{array}
$$


If there exists a subset $K_{0} \subset\left\{k \in K: G_{k}(\bar{x})=0\right\}$ such that

$$
\bar{c}_{i j}(\bar{x})=\sum_{k \in \tilde{K}} \lambda_{k}\left(b_{i j}^{k}-b_{i j(i)}^{k}\right) \geq 0 \quad 1 \leq i \leq n ; \quad j \in J_{i}
$$

whenever $\tilde{K}=\left\{k \in K: G_{k}(\bar{x})>0\right\} \cup K_{0}$, then $\bar{x}$ is efficient for $(G A T P L)$.

Proof. Referring to $\left(\overline{G A T P L}_{\lambda}\right)$ and to the definition of $\tilde{K}$, it follows that if $\bar{s}=$ $\left[\bar{s}_{1}, \ldots, \bar{s}_{k}\right]^{T}$ and $\bar{t}=\left[\bar{t}_{1}, \ldots, \bar{t}_{k}\right]^{T}$ are specified as follows: for all $k, 1 \leq k \leq K$

$$
\bar{s}_{k}=\left\{\begin{array}{ll}
G_{k}(\bar{x}) & \text { if } k \in \tilde{K} \\
0 & \text { otherwise }
\end{array} \quad \bar{t}_{k}= \begin{cases}0 & \text { if } k \in \tilde{K} \\
-G_{k}(\bar{x}) & \text { otherwise }\end{cases}\right.
$$

then $\bar{x}, \bar{s}, \bar{t}$ is a basic feasible solution of $\left(\overline{G A T P L}_{\lambda}\right)$ where the basic variables are $x_{i j(i)}, 1 \leq i \leq n, s_{k}, k \in \tilde{K}$, and $t_{k}, k \in K \backslash \tilde{K}$.

Now, we analyze how to obtain the relative costs of the variables associated with the corresponding basis.

\begin{tabular}{|ccc|ccc|ccc|c|ccc|c|}
\hline$s_{1}$ & $\cdots$ & $s_{K}$ & $t_{1}$ & $\cdots$ & $t_{K}$ & $x_{11}$ & $\cdots$ & $x_{1 m_{1}}$ & $\cdots$ & $x_{n 1}$ & $\cdots$ & $x_{n m_{n}}$ & right hand side \\
\hline \hline-1 & $\cdots$ & 0 & 1 & $\cdots$ & 0 & $b_{11}^{1}$ & $\cdots$ & $b_{1 m_{1}}^{1}$ & $\cdots$ & $b_{n 1}^{1}$ & $\cdots$ & $b_{n m_{n}}^{1}$ & 0 \\
\hline & $\ddots$ & & & $\ddots$ & & $\vdots$ & $\cdots$ & $\vdots$ & $\cdots$ & $\vdots$ & $\cdots$ & $\vdots$ & $\vdots$ \\
\hline 0 & $\cdots$ & -1 & 0 & $\cdots$ & 1 & $b_{11}^{K}$ & $\cdots$ & $b_{1 m_{1}}^{K}$ & $\cdots$ & $b_{n 1}^{K}$ & $\cdots$ & $b_{n m_{n}}^{K}$ & 0 \\
\hline \hline 0 & $\cdots$ & 0 & 0 & $\cdots$ & 0 & 1 & $\cdots$ & 1 & $\cdots$ & 0 & $\cdots$ & 0 & 1 \\
\hline$\vdots$ & $\cdots$ & $\vdots$ & $\vdots$ & $\cdots$ & $\vdots$ & & $\cdots$ & & $\ddots$ & & $\cdots$ & & $\vdots$ \\
\hline 0 & $\cdots$ & 0 & 0 & $\cdots$ & 0 & 0 & $\cdots$ & 0 & $\cdots$ & 1 & $\cdots$ & 1 & 1 \\
\hline \hline$\lambda_{1}$ & $\cdots$ & $\lambda_{K}$ & 0 & $\cdots$ & 0 & 0 & $\cdots$ & 0 & $\cdots$ & 0 & $\cdots$ & 0 & 0 \\
\hline
\end{tabular}

FiguRE 1. Matrix associated with problem $\left(\overline{G A T P L}_{\lambda}\right)$.

To the last row of the tableau in Figure 1, we add each row $k$ multiplied by $\lambda_{k}$ if $k \in \tilde{K}$ (hence the coefficient of $s_{k}$ becomes equal to zero if $k \in \tilde{K}$ ). Furthermore, we substract each row $(K+i)$ multiplied by $\sum_{k \in \tilde{K}} \lambda_{k} b_{i j(i)}^{k}$ for all $i, 1 \leq i \leq n$ (hence the coefficient of $x_{i j(i)}$ becomes equal to zero for all $\left.i, 1 \leq i \leq n\right)$. It is easy to verify that the row vector generated includes the relative costs of the variables, 
and hence

$$
\begin{aligned}
\text { relative cost of } x_{i j}=\bar{c}_{i j}(\bar{x}) & =\sum_{k \in \tilde{K}} \lambda_{k}\left(b_{i j}^{k}-b_{i j(i)}^{k}\right), 1 \leq i \leq n, j \in J_{i} \\
\text { relative cost of } s_{k} & = \begin{cases}0 & \text { if } k \in \tilde{K} \\
\lambda_{k} & \text { otherwise }, 1 \leq k \leq K\end{cases} \\
\text { relative cost of } t_{k} & = \begin{cases}\lambda_{k} & \text { if } k \in \tilde{K} \\
0 & \text { otherwise }, 1 \leq k \leq K .\end{cases}
\end{aligned}
$$

But referring to the hypothesis of the theorem,

$$
\lambda_{k}>0,1 \leq k \leq K, \text { and } \bar{c}_{i j}(\bar{x}) \geq 0,1 \leq i \leq n, j \in J_{i}
$$

Thus the basic feasible solution $\bar{x}, \bar{s}, \bar{t}$ is optimal for $\left(\overline{G A T P L}_{\lambda}\right)$. Furthermore, since $\bar{x}, \bar{s}, \bar{t}$ is a feasible solution of $\left(G A T P L_{\lambda}\right)$ and since $\left(\overline{G A T P L}_{\lambda}\right)$ is a relaxation of $\left(G A T P L_{\lambda}\right)$, it follows that $\bar{x}, \bar{s}, \bar{t}$ is an optimal solution for $\left(G A T P L_{\lambda}\right)$. Hence referring to Geoffrion [6], $\bar{x} \in \mathcal{X}$ is an efficient point of $(G A T P L)$ associated with the weight vector $\lambda>0$.

It is worth noting that Property 3.1 can be derived using the duality theory in multiobjective linear programming introduced in Isermann [7]. Furthermore, the criterion for efficiency in Property 3.1 (i.e., the $\bar{c}_{i j}(\bar{x})$ ) is specified in terms of the violated or binding constraints uniquely. In a sense, these constraints play a role similar to that of the active or binding constraints in single objective optimization.

Unfortunately, the sufficient condition in Property 3.1 is not necessary in general because Geofrion [6] condition is not necessary in general.

\section{LOCAL EFFICIENCY CONDITIONS}

Now, we introduce conditions to characterize the notion of local efficiency with respect to a neighborhood. In the next section, these conditions are shown to be weaker than those that characterize efficient solutions. These conditions have been used (without formally showing that they characterize local efficiency) in Marziliano and Ferland [10] to derive an interactive algorithm to iteratively generate sets of neighboring local efficient solutions. At each iteration the user indicates the solution that he prefers, and this information is used at the next iteration to generate a new set of local efficient solutions. This algorithm is a variant of Zionts and Wallenius [14] approach.

As in Section 3 , let $\bar{x} \in \mathcal{X}$ be such that for all $i, 1 \leq i \leq n$

$$
\begin{aligned}
\bar{x}_{i j(i)}=1 & \text { for some index } j(i) \in J_{i} \\
\bar{x}_{i j}=0 & \text { for all } j \in J_{i}, j \neq j(i) .
\end{aligned}
$$

A solution $x^{\prime} \in \mathcal{X}$ belongs to the neighborhood $\mathcal{N}(\bar{x})$ of $\bar{x}$ if it can be generated from $\bar{x}$ by modifying the assignment of exactly one item. Denote $x^{\prime}=\bar{x} \oplus(i, j)$ 
the solution in $\mathcal{X}$ obtained from $\bar{x}$ by assigning item $i$ to $j$ instead of $j(i)$; i.e.

$$
\begin{aligned}
x_{p q}^{\prime} & =\bar{x}_{p q} \quad 1 \leq p \leq n, p \neq i, q \in J_{p} \\
x_{i q}^{\prime} & =\bar{x}_{i q} \quad q \in J_{i}, q \neq j, j(i) \\
x_{i j}^{\prime} & =1 \\
x_{i j(i)}^{\prime} & =0 .
\end{aligned}
$$

Then

$$
\mathcal{N}(\bar{x})=\left\{x^{\prime} \in \mathcal{X}: \exists(i, j) \text { such that } x^{\prime}=x \oplus(i, j), 1 \leq i \leq n, j \in J_{i}, j \neq j(i)\right\}
$$

The notion of local efficiency with respect to $\mathcal{N}$ can be specified as follows:

$x \in \mathcal{X}$ is locally efficient with respect to $\mathcal{N}$ for $(G A T P L)$ if there is no $z \in \mathcal{N}(x)$ such that

$$
\begin{aligned}
& V_{k}(z) \leq V_{k}(x) \quad 1 \leq k \leq K+1 \\
\text { and } \quad & \\
& V_{\bar{k}}(z)<V_{\bar{k}}(x) \quad \text { for some index } \bar{k}, 1 \leq \bar{k} \leq K .
\end{aligned}
$$

The sufficient conditions for local efficiency are specified in terms of the modification induced on the objective function of $\left(G A T P L_{\lambda}\right)$ if the solution $\bar{x}$ is modified to $x^{\prime}=\bar{x} \oplus(i, j)$ in $\mathcal{N}(\bar{x})$ (i.e., if we move from $\bar{x}$ to the neighboring solution $x^{\prime}$ ). The following technique which measures the modification has been introduced in Mazzola and Neebe [11]. It is easy to verify that the modification $\delta_{i j}^{k}(\bar{x})$ so induced on the function $V_{k}(x)$ is specified as follows:

$\delta_{i j}^{k}(\bar{x})= \begin{cases}b_{i j}^{k}-b_{i j(i)}^{k} & \text { if } G_{k}(\bar{x}) \geq 0 \text { and }\left(b_{i j}^{k}-b_{i j(i)}^{k}\right)>0 \\ \max \left\{0, G_{k}(\bar{x})+b_{i j}^{k}-b_{i j(i)}^{k}\right\} & \text { if } G_{k}(\bar{x})<0 \text { and }\left(b_{i j}^{k}-b_{i j(i)}^{k}\right)>0 \\ \max \left\{-G_{k}(\bar{x}),\left(b_{i j}^{k}-b_{i j(i)}^{k}\right)\right\} & \text { if } G_{k}(\bar{x}) \geq 0 \text { and }\left(b_{i j}^{k}-b_{i j(i)}^{k}\right)<0 \\ 0 & \text { otherwise. }\end{cases}$

The overall modification $\delta_{i j}(\bar{x})$ induced on the objective function of $\left(G A T P L_{\lambda}\right)$ is

$$
\delta_{i j}(\bar{x})=\sum_{k=1}^{K} \lambda_{k} \delta_{i j}^{k}(\bar{x}) .
$$

Theorem 4.1. Consider problem $(G A T P L)$. Let $\lambda>0$ be a weights vector, and $\bar{x} \in \mathcal{X}$ be such that for all $i, 1 \leq i \leq n$

$$
\begin{aligned}
& \bar{x}_{i j(i)}=1 \quad \text { for some index } j(i) \in J_{i} \\
& \text { and } \\
& \bar{x}_{i j}=0 \quad \text { for all } j \in J_{i}, j \neq j(i) .
\end{aligned}
$$


If $\delta_{i j}(\bar{x}) \geq 0,1 \leq i \leq n, j \in J_{i}$, then $\bar{x}$ is locally efficient with respect to $\mathcal{N}$ for $(G A T P L)$.

Proof. For contradiction, assume that $\bar{x}$ is not locally efficient with respect to $\mathcal{N}$. Hence there exists a solution $x^{\prime}=\bar{x} \oplus(i, j)$ in $\mathcal{N}(\bar{x})$ such that

$$
\begin{aligned}
& V_{k}\left(x^{\prime}\right) \leq V_{k}(\bar{x}) \quad 1 \leq k \leq K \\
& V_{\bar{k}}\left(x^{\prime}\right)<V_{\bar{k}}(\bar{x}) \quad \text { for some index } \bar{k}, 1 \leq \bar{k} \leq K .
\end{aligned}
$$

On the one hand, since $V_{\bar{k}}\left(x^{\prime}\right)<V_{\bar{k}}(\bar{x})$, then $G_{\bar{k}}(\bar{x})>0$, and $b_{i j}^{\bar{k}}<b_{i j(i)}^{\bar{k}}$. Hence, referring to the definition of $\delta_{i j}^{\bar{k}}(\bar{x})$, since $G_{\bar{k}}(\bar{x})>0$ and $\left(b_{i j}^{\bar{k}}-b_{i j(i)}^{\bar{k}}\right)<0$ it follows that

$$
\delta_{i j}^{\bar{k}}(\bar{x})=\max \left\{-G_{\bar{k}}(\bar{x}),\left(b_{i j}^{\bar{k}}-b_{i j(i)}^{\bar{k}}\right)\right\}<0 .
$$

On the other hand, for $k \neq \bar{k}$, two cases must be analyzed:

Case 1: $G_{k}(\bar{x}) \geq 0$.

Since $V_{k}\left(x^{\prime}\right) \leq V_{k}(\bar{x})$, it follows that if $G_{k}(\bar{x}) \geq 0$, then $b_{i j}^{k}-b_{i j(i)}^{k} \leq 0$. Furthermore, from the definition of $\delta_{i j}^{k}(\bar{x})$, if $G_{k}(\bar{x}) \geq 0$, then

$$
\delta_{i j}^{k}(\bar{x})= \begin{cases}b_{i j}^{k}-b_{i j(i)}^{k} & \text { if }\left(b_{i j}^{k}-b_{i j(i)}^{k}\right) \geq 0 \\ \max \left\{-G_{k}(\bar{x}),\left(b_{i j}^{k}-b_{i j(i)}^{k}\right)\right\} & \text { otherwise. }\end{cases}
$$

Hence $\delta_{i j}^{k}(\bar{x}) \leq 0$.

Case 2: $G_{k}(\bar{x})<0$.

Since $V_{k}\left(x^{\prime}\right) \leq V_{k}(\bar{x})$, it follows that if $G_{k}(\bar{x})<0$, then $G_{k}\left(x^{\prime}\right)=G_{k}(\bar{x})+\left(b_{i j}^{k}-\right.$ $\left.b_{i j(i)}^{k}\right) \leq 0$. Furthermore, from the definition of $\delta_{i j}^{k}(\bar{x})$, if $G_{k}(\bar{x})<0$, then

$$
\delta_{i j}^{k}(\bar{x})= \begin{cases}\max \left\{0, G_{k}(\bar{x})+\left(b_{i j}^{k}-b_{i j(i)}^{k}\right)\right\} & \text { if }\left(b_{i j}^{k}-b_{i j(i)}^{k}\right)>0 \\ 0 & \text { otherwise. }\end{cases}
$$

Hence $\delta_{i j}^{k}(\bar{x}) \leq 0$ in this case also.

Finally, since $\lambda>0$, it follows that

$$
\delta_{i j}(\bar{x})=\sum_{k=1}^{K} \lambda_{k} \delta_{i j}^{k}(\bar{x})<0
$$

a contradiction. Thus, $\bar{x}$ is locally efficient with respect to $\mathcal{N}$ for $(G A T P L)$.

At each iteration of the method introduced in Marziliano and Ferland [10], the authors rely on the result in Theorem 4.1 to determine how to modify each 
$\lambda_{k}, 1 \leq k \leq K$, individually in order to generate a new local efficient point in the neighborhood of the current local efficient point. Hence a set of new local efficient points in the neighborhood of the current efficient point together with their associated weight vector $\lambda$ are generated at each iteration.

Unfortunately, the sufficient condition in Theorem 4.1 is not necessary. Indeed, consider the $(G A T P L)$ where

$$
\begin{aligned}
& G_{1}(x)=10 x_{11}+2 x_{12}+x_{21}+11 x_{22} \\
& G_{2}(x)=x_{11}+2 x_{12}+10 x_{21}+8 x_{22} \\
& G_{3}(x)=-x_{11}-x_{12}-x_{21}-x_{22}
\end{aligned}
$$

and $\mathcal{X}$ is the set of points in $\mathbb{R}^{4}$ such that

$$
\begin{aligned}
& x_{11}+x_{12}=1 \\
& x_{21}+x_{22}=1 \\
& x_{i j}=0 \text { or } 1, \quad 1 \leq i \leq 2,1 \leq j \leq 2 .
\end{aligned}
$$

There exist four feasible solutions in $\mathcal{X}$ :

$$
x^{1}=[1,0,1,0], x^{2}=[1,0,0,1], x^{3}=[0,1,1,0] \text {, and } x^{4}=[0,1,0,1] .
$$

The corresponding values of the $G_{k}(x), 1 \leq k \leq 3$ are summarized Table 1 .

TABLE 1 . Values of the $G_{k}(x)$.

\begin{tabular}{r|cccc}
$x$ & {$[1,0,1,0]$} & {$[1,0,0,1]$} & {$[0,1,1,0]$} & {$[0,1,0,1]$} \\
\hline$G_{1}(x)$ & 11 & 21 & 3 & 13 \\
$G_{2}(x)$ & 11 & 9 & 12 & 10 \\
$G_{3}(x)$ & -2 & -2 & -2 & -2
\end{tabular}

It is easy to verify that $x^{1}=[1,0,1,0]$ is locally efficient with respect to $\mathcal{N}$. But there is no vector $\lambda=\left[\lambda_{1}, \lambda_{2}, \lambda_{3}\right]^{T}>0$ such that $\delta_{i j}\left(x^{1}\right) \geq 0,1 \leq i \leq 2$, 
$1 \leq j \leq 2$. Indeed, for contradiction, assume otherwise. Since

$$
\begin{array}{lll}
\delta_{12}^{1}\left(x^{1}\right)=-8, & \delta_{12}^{2}\left(x^{1}\right)=1, & \delta_{12}^{3}\left(x^{1}\right)=0 \\
\delta_{22}^{1}\left(x^{1}\right)=10, & \delta_{22}^{2}\left(x^{1}\right)=-2, & \delta_{22}^{3}\left(x^{1}\right)=0,
\end{array}
$$

and hence $16 \lambda_{1} \leq 2 \lambda_{2} \leq 10 \lambda_{1}$ implies the $\lambda_{1} \leq 0$, a contradiction.

\section{LOCAL EFFICIENCY vs. EFFICIENCY}

Referring to the definitions, it is trivial that an efficient point is a local efficient point. As one can expect, it is easy to verify that whenever $\bar{x}$ satisfies the sufficient condition of Property 3.1, then it satisfies the sufficient condition for local efficiency given in Theorem 4.1. This result is shown in the following theorem.

Theorem 5.1. Consider problem $(G A T P L)$. Let $\lambda>0$ be a weights vector, and $\bar{x} \in \mathcal{X}$ be such that for all $i, 1 \leq i \leq n$

$$
\begin{aligned}
\bar{x}_{i j(i)}=1 & \text { for some index } j(i) \in J_{i} \\
\bar{x}_{i j}=0 & \text { for all } j \in J_{i}, j \neq j(i) .
\end{aligned}
$$

Denote $\tilde{K}=\left\{k \in K: G_{k}(\bar{x})>0\right\} \cup K_{0}$, where $K_{0} \subset\left\{k: G_{k}(\bar{x})=0\right\}$. If $\bar{c}_{i j}(\bar{x})=\sum_{k \in \bar{K}} \lambda_{k}\left(b_{i j}^{k}-b_{i j(i)}^{k}\right) \geq 0,1 \leq i \leq n, j \in J_{i}$, then $\left.\delta_{i j}(\bar{x})\right) \geq 0,1 \leq i \leq n$, $j \in J_{i}$.

Proof. Denote $\bar{c}_{i j}^{k}(\bar{x})=b_{i j}^{k}-b_{i j(i)}^{k}, 1 \leq i \leq n, j \in J_{i}, 1 \leq k \leq K$. By definition

$$
\bar{c}_{i j}(\bar{x})=\sum_{k \in \tilde{K}} \lambda_{k}\left(b_{i j}^{k}-b_{i j(i)}^{k}\right)
$$

For any $k \in\left\{k \in K: G_{k}(\bar{x})>0\right\}$,

i) if $\left(b_{i j}^{k}-b_{i j(i)}^{k}\right) \geq 0$, then $\delta_{i j}^{k}(\bar{x})=b_{i j}^{k}-b_{i j(i)}^{k}=\bar{c}_{i j}^{k}(\bar{x})$;

ii) if $\left(b_{i j}^{k}-b_{i j(i)}^{k}\right)<0$, then

$$
\begin{aligned}
\delta_{i j}^{k}(\bar{x}) & =\max \left\{-G_{k}(\bar{x}),\left(b_{i j}^{k}-b_{i j(i)}^{k}\right)\right\} \\
& =\max \left\{-G_{k}(\bar{x}), \bar{c}_{i j}^{k}(\bar{x})\right\} \\
& \geq \bar{c}_{i j}^{k}(\bar{x}) .
\end{aligned}
$$

Similarly, for any $k \in\left\{k \in K: G_{k}(\bar{x})=0\right\}$,

i) if $\left(b_{i j}^{k}-b_{i j(i)}^{k}\right) \geq 0$, then $\delta_{i j}^{k}=\bar{c}_{i j}^{k}(\bar{x}) \geq 0$;

ii) if $\left(b_{i j}^{k}-b_{i j(i)}^{k}\right)<0$, then $\delta_{i j}^{k}=\max \left\{0, \bar{c}_{i j}^{k}(\bar{x})\right\}=0$.

It follows that

$$
\sum_{k \in \tilde{K}} \lambda_{k} \delta_{i j}^{k}(\bar{x}) \geq \sum_{k \in \tilde{K}} \lambda_{k} \bar{c}_{i j}^{k}(\bar{x})
$$


Finally, for any $k \in\left\{k \in K: G_{k}(\bar{x})<0\right\}$,

$$
\delta_{i j}^{k}(\bar{x})= \begin{cases}\max \left\{0, G_{k}(\bar{x})+\left(b_{i j}^{k}-b_{i j(i)}^{k}\right)\right\} & \text { if }\left(b_{i j}^{k}-b_{i j(i)}^{k}\right)>0 \\ 0 & \text { otherwise. }\end{cases}
$$

Thus, $\delta_{i j}^{k}(\bar{x}) \geq 0$, and consequently

$$
\sum_{k \notin \tilde{K}} \lambda_{k} \delta_{i j}^{k}(\bar{x}) \geq 0
$$

Using relations (1) and (2), it follows that

$$
\delta_{i j}(\bar{x})=\sum_{k \in \tilde{K}} \lambda_{k} \delta_{i j}^{k}(\bar{x})+\sum_{k \notin \tilde{K}} \lambda_{k} \delta_{i j}^{k}(\bar{x}) \geq \sum_{k \in \tilde{K}} \lambda_{k} \bar{c}_{i j}^{k}(\bar{x})=\bar{c}_{i j}(\bar{x}) .
$$

Hence $\bar{c}_{i j}(\bar{x}) \geq 0,1 \leq i \leq n, j \in J_{i}$, implies that $\delta_{i j}(\bar{x}) \geq 0,1 \leq i \leq n, j \in J_{i}$.

\section{CONCLUding REMARKS}

It is quite straighforward to extend these results to deal with the generalized version of $(A T P)$ where the set $\mathcal{X}$ is replaced by $\mathcal{X}(a)$

$$
\mathcal{X}(a)=\left\{x: \sum_{j \in J_{i}} x_{i j}=a_{i}, 1 \leq i \leq n ; x_{i j}=0 \text { or } 1,1 \leq i \leq n, j \in J_{i}\right\}
$$

and $a=\left[a_{1}, a_{2}, \ldots, a_{n}\right]^{T}, a_{i} \geq 1$ and integer, $1 \leq i \leq n$. In this case, the constraint $\sum_{j \in J_{i}} x_{i j}=1$ in $\left(G A T P L_{\lambda}\right)$ is replaced by $\sum_{j \in J_{i}} x_{i j}=a_{i}, 1 \leq i \leq n$. Hence, the condition is derived by referring to the optimal condition of the corresponding relaxed problem $\overline{\left(G A T P L_{\lambda}\right)}$ obtained by relaxing the integrality conditions on the variables $x_{i j} ;$ i.e. the constraints $x_{i j}=0$ or 1 are replaced by $x_{i j} \geq 0$ and $x_{i j} \leq 1$, $1 \leq i \leq n, j \in J_{i}$. They are summarized in the following theorems.

Theorem 6.1. Consider problem $(G A T P L)$ where $\mathcal{X}$ is replaced by $\mathcal{X}(a)$. Let $\lambda>0$ be a weights vector, and $\bar{x} \in \mathcal{X}(a)$ such that for all $i, 1 \leq i \leq n$, there exists a subset $J(i) \subset J_{i}$ of cardinality $a_{i}$, and

$$
\begin{aligned}
& \bar{x}_{i j}=1 \quad j \in J(i) \\
& \bar{x}_{i j}=0 \quad j \in J_{i}, j \notin J(i) .
\end{aligned}
$$

If there exists a subset $K_{0} \subset\left\{k \in K: G_{k}(\bar{x})=0\right\}$ such that for all $i, 1 \leq i \leq n$, 
there exists an index $j(i) \in J(i)$ such that

$$
\bar{c}_{i j}(\bar{x})=\sum_{k \in \tilde{K}} \lambda_{k}\left(b_{i j}^{k}-b_{i j(i)}^{k}\right)\left\{\begin{array}{lll}
\geq 0 & \text { if } & j \notin J(i) \\
\leq 0 & \text { if } & j \in J(i),
\end{array}\right.
$$

whenever $\tilde{K}=\left\{k \in K: G_{k}(\bar{x})>0\right\} \cup K_{0}$, then $\bar{x}$ is efficient for the problem.

The argument is similar to the proof of Property 3.1 We refer to the variant of the simplex algorithm where upper bounds are specified for the variables, and we use the basic solution where the variables $x_{i j(i)}, 1 \leq i \leq n, s_{k}, k \in \tilde{K}$, and $t_{k}, k \notin \tilde{K}$, are basic.

The local efficiency is specified with respect to the following neighborhood $\overline{\mathcal{N}}$. A solution $x^{\prime} \in \mathcal{X}(a)$ belongs to the neighborhood $\overline{\mathcal{N}}(\bar{x})$ if it can be generated from $\bar{x}$ by modifying exactly one assignment of exactly one item. If $J(i)$ denotes the subset of indices in $J_{i}$ such that $x_{i j}=1$ if $j \in J(i)$, then denote $x^{\prime}=\bar{x} \oplus(i, \bar{j}, j)$ the solution in $\mathcal{X}(a)$ obtained from $\bar{x}$ by modifying the assignment of item $i$ from $\bar{j} \in J(i)$ to $j \notin J(i) ;$ i.e.

$$
\begin{aligned}
& x_{p q}^{\prime}=\bar{x}_{p q} \quad 1 \leq p \leq n, p \neq i, q \in J_{p} \\
& x_{i q}^{\prime}=\bar{x}_{i q} \quad q \in J_{i}, q \neq j, \bar{j} \\
& x_{i j}^{\prime}=1 \\
& x_{i \bar{j}}^{\prime}=0 .
\end{aligned}
$$

Then

$$
\begin{aligned}
\overline{\mathcal{N}}(\bar{x})=\left\{x^{\prime} \in \mathcal{X}(a): \exists \quad\right. & \text { a tuplet }(i, \bar{j}, j), 1 \leq i \leq n, \bar{j} \in J(i), j \notin J(i) \\
& \text { such that } \left.x^{\prime}=\bar{x} \oplus(i, \bar{j}, j)\right\} .
\end{aligned}
$$

Theorem 6.2. Consider problem $(G A T P L)$ where $\mathcal{X}$ is replaced by $\mathcal{X}(a)$. Let $\lambda>0$ be a weights vector, and $\bar{x} \in \mathcal{X}(a)$ such that for all $i, 1 \leq i \leq n$, there exists a subset $J(i) \subset J_{i}$ of cardinality $a_{i}$, and

$$
\begin{array}{ll}
\bar{x}_{i j}=1 & j \in J(i) \\
\bar{x}_{i j}=0 & j \notin J(i) .
\end{array}
$$

If for all $i, 1 \leq i \leq n$, there exists an index $j(i) \in J(i)$ such that

$$
\delta_{i j}(\bar{x}) \begin{cases}\geq 0 & \text { if } j \notin J(i) \\ \leq 0 & \text { if } j \in J(i)\end{cases}
$$

then $\bar{x}$ is locally efficient with respect to $\overline{\mathcal{N}}$ for the problem.

In Marziliano and Ferland [10], the authors introduce a variant of the interactive method of Zionts and Wallenius [14]. At each iteration, given a local efficient 
point $\bar{x}$, a set of neighboring local efficient points belonging to $\mathcal{N}(\bar{x})$ (to $\overline{\mathcal{N}}(\bar{x})$, respectively) are generated according to the criterion introduced in Theorem 4.1 (in Th. 6.2, respectively). When the method satisfies the stopping criteria, the result in Property 3.1 (in Th. 6.1, respectively) can be used to verify if the current local efficient point is in fact efficient.

Furthermore, this variant could be easily modified in order to generate at each iteration a set of neighbouring efficient points belonging to $\mathcal{N}(\bar{x})$ (to $\overline{\mathcal{N}}(\bar{x})$, respectively) according to the criterion introduced in Property 3.1 (in Th. 6.1, respectively).

\section{REFERENCES}

[1] D. Costa, Méthodes de résolution constructives, séquentielles et évolutives pour des problèmes d'affectation sous-contraintes, Doctoral dissertation. Mathematics Department, École Polytechnique Fédérale de Lausanne, Switzerland (1995).

[2] J.A. Ferland, Generalized Assignment Type Problems, a Powerful Modeling Scheme, in Practice and Theory of Automated Timetabling II, edited by E. Burke and M. Carter. Springer, Lecture Notes in Comput. Sci. 1408 (1998) 53-77.

[3] J.A. Ferland, I. Berrada, I. Nabli, A. Ahiot, P. Michelon and V. Gascon, Generalized Assignment-Type Goal Programming Problem and Application to Nurse Scheduling. J. Heuristics 7 (2001) 391-413.

[4] J.A. Ferland, A. Hertz and A. Lavoie, An Objected Oriented Methodology For Solving Assignment Type Problems With Neighborhood Search Techniques. Oper. Res. 44 (1996) 347-359.

[5] J.A. Ferland and A. Lavoie, Exchanges Procedures For Timetabling Problems. Discrete Appl. Math. 35 (1992) 237-253.

[6] A.M. Geoffrion, Proper Efficiency and Theory of Vector Maximization. J. Math. Anal. Appl. 22 (1968) 618-630.

[7] H. Isermann, The Relevance of Duality in Multiple Objective Linear Programming. TIMS Studies in the Management Sci. 6 (1977) 241-262.

[8] F.A. Lootsma, Optimization with Multiple Objectives, in Mathematical Programming: Recent Developments and Applications, edited by M. Iri and K. Tanabe (1989) 333-364.

[9] P. Marziliano, Problèmes multicritères avec contraintes d'affectation, Master Thesis. Département d'Informatique et de Rechereche Opérationnelle, Université de Montréal, Canada (1996).

[10] P. Marziliano and J.A. Ferland, A Heuristic Approach for Multiobjective Problems with Assignment Constraints, Publication \# 1128. Département d'Informatique et de Recherche Opérationnelle, Université de Montréal, Canada (1998).

[11] J.B. Mazzola and A.W. Neebe, Resource-Constrained Assignment Scheduling. Oper. Res. 34 (1986) 560-572

[12] V. Robert, La confection d'horaires par décomposition en sous-problèmes d'affectation, Doctoral dissertation. Mathematics Department, École Polytechnique Fédérale de Lausanne, Switzerland (1996).

[13] R.E. Steuer, Multiple Criteria Optimization: Theory,Computation and Application. Wiley, New York (1986).

[14] S. Zionts and J. Wallenius, An Interactive Multiple Objective Linear Programming Method for a Class of Underlying Nonlinear Utility Functions. Management Sci. 29 (1983) 519-529. 